\title{
Accuracy responses in species identification varying DNA barcode lengths with a Naïve Bayes classifier: Efficacy of mini-barcode under a supervised machine learning approach
}

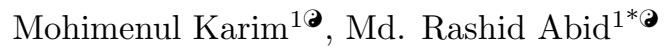 \\ 1 Department of Computer Science and Engineering, Bangladesh University of \\ Engineering and Technology, Dhaka, Bangladesh \\ (2These authors contributed equally to this work. \\ * 1018052031@grad.cse.buet.ac.bd
}

\begin{abstract}
Specific gene regions in DNA, like COI (Cytochrome c oxidase I) in case of animals, have been defined as DNA barcode and many studies proved that it can be used as an identifier to distinguish species. The standard length of a DNA barcode is approximately $650 \mathrm{bp}$. But because of the challenges in sequencing technologies or unavailability of high-quality genomic DNA, it is not always possible to get the full barcode sequence of an organism. As a result, recent studies suggest that mini-barcodes can provide a good contribution in the species identification process. Among various methods proposed for the identification task, supervised machine learning methods have been shown effective. In this study, we have analyzed the effect of different barcode lengths on species identification from the perspective of supervised machine learning and suggested a general approximation of required length of mini-barcode in this regard. We have implemented a Naïve Bayes classifier as our model and implied the effectiveness of mini-barcode by demonstrating the accuracy responses varying the length of DNA barcode sequences.
\end{abstract}

\section{Introduction}

Species identification is naturally an elementary part in biological researches. According to the Catalogue of Life $(\mathrm{CoL})$, which is a hierarchically ranked system based on expert and coherent opinions provided by taxonomists, more than 1.6 million species have been identified throughout the world so far [1]. Still, the total number of species is estimated to be 8.7 millions and it is suggested that in spite of over 250 years of taxonomic classification, the existing records tend to cover only a small portion of species on earth $(14 \%)$ and in the ocean $(9 \%)$ while the rest are yet to be described [2]. From the taxonomic point of view, the process of species discovery can be said to be broadly comprised of three pivotal but onerous steps: specimen collection, species level sorting and lastly species identification/description 3. In addition to being already exigent by virtue of their own nature of work and thereby creating taxonomic impediments, these steps bring morphological characteristics into account in the traditional 'evidence-based' taxonomic approach and thus inflict likewise challenges in the decision making process [4]. For instance, in the specimen collection phase due to adverse situations often organism-fragments, stomach contents, feces, saliva, skin-scraps, blood, pollen etc can be achievable as samples which lack substantial morphological characteristics. 
Additionally, in case of archival museum specimens, distinguishing morphological details are often absent. Also in the next phase of species level sorting, it is observed that many congeneric species possess morphological differences so negligible that they are often misclassified as a single species. Furthermore, in cases of species-complex and cryptic species, morphological differences are almost indiscernible. It might also be the case that due to sexual dimorphism, two sexes of same species can exhibit significantly disparate morphological attributes misleadingly appearing to be of different species. Various organisms can also develop conspicuous morphological changes in different stages of their life-cycle.

For addressing all the aforementioned issues, a more advanced, accurate, fast and efficient alternative approach in taxonomy was long yearned for which would attenuate morphological subservience in such cases. It is deemed that microgenomic approach involving DNA sequences has been successfully established as an extremely promising technique in recent years for species identification in this regard [5]. In this technique, a short portion of DNA from a specific gene or genes of an organism is used to uniquely identify a species in comparison with a reference library 6 . That specific portion is referred to as DNA barcode of that species as the process is similar to the process of identifying retail products with the help of machine readable barcodes. Different gene regions are used as DNA barcodes for different groups, namely: cytochrome-c-oxidase-I (COI/COX1) gene found in mtDNA for animals 7], rbcL and matK for plants [8] and the Internal Transcribed Spacer (ITS) rRNA for fungi 9]. Species identification with DNA Barcode can be treated as a classification problem where an unknown DNA barcode sequence is matched against a reference library comprising of DNA barcode sequences of already known species [10. Various methods have been proposed for addressing this problem [11] and supervised machine learning approach is a prominent one among them where the reference dataset of known species is used as Train Set for training corresponding machine learning models and barcode sequences requiring classifications are placed in Test Set for query [12].

Notwithstanding the prominence of DNA barcode in species identification, several shortcomings are however perceived in some specific cases. Although PCR amplification and sequencing is usually consistent in freshly collected and well-preserved specimens, obtaining a full-length $(\sim 650 \mathrm{bp})$ barcode in case of archival museum specimens which are preserved under sub-optimal or DNA-unfriendly conditions for years is often difficult [13]. This is also the case for processed biological materials (e.g. food products, pharmaceuticals etc.) and decayed tissues due to their degraded quality of DNA [14]. In such instances where obtaining a full-length barcode is hard, shorter barcode sequences ( 100-300 bp), often referred to as Mini-barcodes, have been found efficacious for species-level identification owing to their comparatively better amplification gain [15]. For this reason, mini-barcode is also being popularly used in DNA metabarcoding strategies based on environmental DNA (eDNA) [16] and researching ecological biodiversity [17. Mini-barcodes can also be advantageously applied in alternative next generation short-read sequencing platforms providing higher throughput but remaining more cost-effective at the same time 13 .

Since DNA Barcode was exercised as an effective molecular approach in species diagnosis by Hebert et al. 6], it has been used as a robust viewpoint in a wide range of studies 18 20]. iBOL (International Barcode of Life) and CBOL (The Consortium for the Barcode of Life) has also patronized the development initiatives for establishing DNA Barcode as a global standard for species identification [21. Methodologies perceived in the literature review of species identification approaches with DNA barcoding can be divided into few major categories 11] 22] 23]. In Distance Based approaches, the reference library sequences are ranked with respect to their distances with the query barcode sequence on basis of the impression that small distances suggest 
two barcodes might be of specimens of same species while large distances evince their belonging to two different species. This distance, commonly referred to as Barcode Gap [24], might be calculated on the basis of pairwise p-distances [6] or K2P distance [22]. TaxI 25] uses pairwise distances as distance measures whereas BOLD 26] uses K2P distances by default. In Similarity Based approaches, the query sequences are usually assigned to species based on a Similarity score 27 indicating how much barcode characters they have in common. BLAST [28, FASTA 29], TaxonDNA [30], $N N$ [22] are some of the prominent examples in this regard. Phylogenetic Approaches construct trees using hierarchical clustering 27 where distinct clusters represent distinct species [31]. It can adopt NJ 32], Parsimony (PAR [33]), Maximum Likelihood (PhyML 34]) or Bayesian Inference (MRBAYES [35]) as underlying methods. Statistical Approaches use population genetics assumptions 36 based on coalescent theory (GMYC [37]) which takes phylogenetic uncertainty (SAP-NJ [38]), likelihood ratio tests [39] etc. into account. Character Based approaches investigate the presence/absence of particular characters, known as Diagnostic Sites, instead of total sequence 23 imitating morphological approaches. CAOS [40], $B L O G$ [10, DNABar 41], BRONX [27], DOME-ID 27] are some instances of this approach. There are also some Alignment-Free tools such as: ATIM-TNT (Tree-based) 27], CVTreeAlpha1.0 (Component Vector based) [42], Spectrum Kernel [43], Alfie (python based) 44] as well as Web Based Tools such as: Linker [45, iBarcode [46], BioBarcode [47], ConFind 48 for DNA barcoding. Comparative analysis of these major classical barcoding approaches are also extensively studied [11 [27] but there is no general consensus about a single best method to analyze DNA barcode data in species identification 22] 23 .

Machine learning methods have been introduced as state-of-the-art methods for their extensive applicability in species identification with DNA Barcoding [12]. Although supervised statistical classification methods (CART, RF and Kernel methods) were studied previously [22; supervised machine learning algorithms e.g. SVM, Jrip, J48 (C4.5), Naïve Bayes were compared on WEKA platforms against those ad-hoc methods by Weitschek et al. and it was observed that SVM and Naïve Bayes outperform on average in both synthetic and empirical datasets 12. Simple-logistic, IBK, PART, Attribute-selected Classifier, Bagging approaches were also implemented in another study [49] in this regard. SMO, BP-NN [50], RF 51], k-mer based approaches [52] [53] can also be perceived in recent studies. Naïve Bayes is also applied on COI 54 barcode database demonstrating misclassification rates and also on ribosomal databases 55 effectively.

With the exploration of the prospects of DNA minibarcode in species identification [56], the application of DNA barcode has also significantly broadened. It is observed that although full length barcodes perform best with $97 \%$ species resolution, $90 \%$ and $95 \%$ identification success can be obtained with 100bp and $250 \mathrm{bp}$ mini barcodes respectively 15. In case of species diagnosis from damaged/degraded DNA samples, $\sim 100-300 \mathrm{bp}$ mini-barcodes have also shown promising results [14]. Tree-based methods, objective clustering, automatic barcode gap discovery (ABGD), Poisson Tree Process (PTP) were also examined for analyzing the utility of minibarcodes for species identification [3]. Although no simple straight-forward formula is available through which necessary sequence lengths for ensuring species identification can be easily predicted [7], the general assumption of achieving superior performance by using full barcode length can be questioned as a saturation point is often seen beyond which the additional length-data create only a little impact in comparison to the increased cost overheads [3]. In this work of ours, we have tried to shed some lights on this question from the machine learning perspective by demonstrating the accuracy responses of species identification at different partial barcode lengths after implementing a Naïve 


\section{Materials and methods}

\section{3}

\section{Datasets}

\section{Empirical Data}

Weitschek et al. stated that for species identification, a full reference set with all possible nucleotide polymorphisms for the sequences of each species is required as well as a sufficient reference set is important to avoid over-fitting or under-fitting [12. For this purpose, we included the datasets they used in their work of 12 in our analysis which is basically a collection of published empirical datasets and simulated DNA barcode datasets. The public empirical datasets of Bats, Birds, Cypraeidae, Drosophila and Fishes were chosen from GenBank Nucleotide Database for high phylogenetic diversity and were available for download at dmb.iasi.cnr.it/supbarcodes.php in FASTA format 12. The datasets referred here as Bird2, Fish2 and Butterfly2 were extracted from BOLD and available in CSV format at http://dimacs.rutgers.edu/archive/Workshops/BarcodeDataSets/. Another dataset was considered while performing our analysis, which appears to be the first large-scale genetic assessment of the butterflies of Southern South America (available for download at dx.doi.org/10.5883/DS-BUNEACAR) highlighting several cases of both deep intraspecific and shallow interspecific divergence [57]. COI gene is used as gene region in DNA barcode sequences of all these datasets. The detailed overview of these empirical datasets having COI gene as barcode sequence can be found in Table 1 .

Table 1. Summary of The Empirical Datasets: COI Gene as Barcodes

\begin{tabular}{|c|c|c|c|c|c|}
\hline Dataset & No of sequences $\mid$ & $\mid$ Sequence Length $\mid$ & $\mid$ No of Species $\mid$ & | Gene Region $\mid$ & Ref \\
\hline Cypraeidae & $\mid 2,008$ & 614 & 211 & COI & \\
\hline Drosophila & 615 & 663 & 19 & COI & 5 \\
\hline Bats & 826 & 659 & 82 & COI & \\
\hline Fishes & 626 & 702 & 82 & COI & $\overline{\overline{6}}$ \\
\hline Birds & 1,700 & 691 & 150 & $\mathrm{COI}$ & 61 \\
\hline Bird 2 & 2589 & 990 & 656 & COI & 6 \\
\hline Fish 2 & 754 & 901 & 211 & COI & 6 \\
\hline Butterfly & 2028 & 658 & 417 & COI & 5 \\
\hline Butterfly 2 & 4267 & 901 & 561 & COI & \\
\hline
\end{tabular}

With a view to inspecting the overall scenario of species identification using DNA Barcoding and analyzing the accuracy responses with varying barcode lengths, we incorporated datasets of plants and fungi as well. These empirical datasets use rbcL and ITS gene regions respectively and are available for download at dmb.iasi.cnr.it/supbarcodes.php in FASTA format 12. The detailed overview of these empirical datasets of plants and fungi can be found in Table 2

Table 2. Summary of The Empirical Datasets of Fungi, Inga and Algae

\begin{tabular}{|c|c|c|c|c|c|}
\hline Dataset & No of sequences & | Sequence Length & $\mid$ No of Species $\mid$ & | Gene Region $\mid$ & Ref \\
\hline Fungi & 50 & $\mid 930$ & 8 & ITS & 9 \\
\hline Inga & 913 & 1,838 & 56 & ITS & 64] \\
\hline Algae & 26 & 1,128 & 5 & rbcL & 8 \\
\hline
\end{tabular}




\section{Simulated Data}

We have also used simulated real DNA Barcode datasets in our work for further analysis. These datasets were also available for downlaod at dmb.iasi.cnr.it/supbarcodes.php in FASTA format 12. As stated in [11, these data were simulated considering time of species divergence and the effective population size $\left(N_{e}\right) . N_{e}=1000,10000,50000$ were used respectively in simulating gene trees and the dataset complexity was increased with population size. After that, DNA barcode sequences were simulated on the additive gene trees with 650 base sequence-length bearing similarity to the real-life size of a standard DNA barcode. Each dataset consists of 50 species and 20 individuals per species. The detailed overview of simulated datasets is given in Table 3 .

Table 3. Summary of The Simulated Datasets

\begin{tabular}{|c|c|c|c|c|c|}
\hline Dataset & $\mid \mathbf{N}_{\mathrm{e}}$ & No of Individual & | Sequence Length & $\mid$ No of Species $\mid$ & Ref \\
\hline Ne1000 & 1000 & 20 & 650 & 50 & 111 \\
\hline Ne10000 & 10000 & 20 & 650 & 50 & 11 \\
\hline Ne50000 & 50000 & 20 & 650 & 50 & $\overline{11}$ \\
\hline
\end{tabular}

\section{Methodology}

\section{Naïve Bayes}

Naïve Bayes 65 is one of those methods which are often used when a large reference set is available. Naive Bayes is based on Bayes' Theorem and it is assumed that the predictors are independent of each other. If we have an instance $\mathrm{S}$ with $\mathrm{n}$ features which is represented by a feature vector $x=\left\{x_{1}, x_{2}, \ldots, x_{n}\right\}$, this model assigns to $\mathrm{S}$ a probability $P\left(C_{m} \mid x_{1}, x_{2}, \ldots, x_{n}\right)$. Here, $C_{m}$ is the $m^{\text {th }}$ class among all possible classes. Using Bayes' theorem, we can calculate the posterior probability $P\left(C_{m} \mid x\right)$ from likelihood $P\left(x \mid C_{m}\right)$, prior probability $P\left(C_{m}\right)$ and evidence $P(x)$.

$$
P\left(C_{m} \mid x\right)=\frac{P\left(x \mid C_{m}\right) P\left(C_{m}\right)}{P(x)}
$$

Under naïve conditional assumption,

$$
P\left(x_{i} \mid x_{i+1}, \ldots, x_{n}, C_{m}\right)=P\left(x_{i} \mid C_{m}\right)
$$

So the posterior probability can be calculated as follows.

$$
\frac{P\left(C_{m}\right) \prod_{i=1}^{n} P\left(x_{i} \mid C_{m}\right)}{P(x)}
$$

As $\mathrm{P}(\mathrm{x})$ is constant for given input, we can only consider the numerator.

Using the Naïve Bayes model, the classification rule can be implemented as follows, where $\hat{y}$ is the class label.

$$
\hat{y}=\underset{m}{\operatorname{argmax}} P\left(C_{m}\right) \prod_{i=1}^{n} P\left(x_{i} \mid C_{m}\right)
$$

\section{Species Identification Using Naïve Bayes}

Because of the efficacy of Naïve Bayes Algorithm in supervised prediction problem 54, we have used this model for species identification process. We can outline the identification approach as follows: 
Let, $b_{k}$ is the $k^{t h}$ barcode sequence in the dataset where $k=1,2, \ldots, n_{b}$ and $n_{b}=$ no of barcode entries in the dataset. $x_{1 k}, x_{2 k}, \ldots, x_{n k}$ are the nucleotides in a barcode sequence $b_{k}$ of length $\mathrm{n}$. If $C_{p}$ is the $p^{t h}$ species among different classes of species, the probability that the barcode sequence $b_{k}$ belongs to the species $C_{p}$ is:

$$
P\left(C_{p} \mid b_{k}\right)=\frac{P\left(b_{k} \mid C_{p}\right) P\left(C_{p}\right)}{P\left(b_{k}\right)}
$$

The prior probability in eq. 5 can be calculated as follows:

$$
\begin{aligned}
P\left(b_{k} \mid C_{p}\right) & =P\left(x_{1 k}, x_{2 k}, \ldots, x_{n k} \mid C_{p}\right) \\
& =P\left(x_{1 k} \mid x_{2 k}, \ldots, x_{n k}, C_{p}\right) P\left(x_{2 k} \mid x_{3 k}, \ldots, x_{n k}, C_{p}\right) \\
& \ldots P\left(x_{n k} \mid C_{p}\right) \\
& =P\left(x_{1 k} \mid C_{p}\right) P\left(x_{2 k} \mid C_{p}\right) \ldots P\left(x_{n k} \mid C_{p}\right)
\end{aligned}
$$

In the above equation, $x_{i k}$ refers to the nucleotide base at $i^{\text {th }}$ position of $k^{\text {th }}$ sequence in the dataset and $P\left(x_{i k} \mid C_{p}\right)$ means the probability of occurring nucleotide base $x_{i k}$ in species $C_{p}$ at position $i$ where $i=1, \ldots, n$. So, if $N_{c_{p}}$ and $N_{x_{i k}}$ are the number of samples of $C_{p}$ and the number of occurrences of $x_{i k}$ at position $i$ respectively, then we calculate this probability as:

$$
P\left(x_{i k} \mid C_{p}\right)=\frac{N_{x_{i k}}}{N_{C_{p}}}
$$

If the proportion is equal to 0 , then the posterior probability will become 0 . To avoid this, we replaced zero with a very small fractional value $\alpha$, where $0<\alpha<<1$. This $\alpha$ can be considered as the mutation rate of nucleotides in the COI region [54].

$\alpha=9.7 \times 10^{-8}$ was proposed as the estimated value of this mutation rate in COI genes 66] and hence is also used here. In case of ambiguous base (e.g., $N$ ) or missing data (e.g., -) at any position, we have not considered those positions for identification purpose. In some datasets, there is a significant number of missing data (i.e., -) in a significant number of sequences because of sequence alignments. Ignoring these missing data in such cases can affect the performance of our model in identification process. For this reason, in these situations we replaced a missing value in a particular position with the most frequently occurring nucleotide in that position within a species.

\section{Data Preparation}

The empirical and simulated DNA Barcode datasets discussed in 'Datasets' segment are used to perform our analysis. Most of these empirical datasets were already split into $80 \%$ and $20 \%$ sequences per species in training and testing set respectively by biologists maintaining necessary polymorphism and species divergences [11]. In cases where only a single dataset file was provided, it was split into Train and Test set on the basis of $80 \%-20 \%$ random split. But it was ensured that whenever possible, each dataset consists 4 or more representing sequences per species in the training set as it was found necessary for obtaining a reliable classification performance [12]. The simulated datasets were also split into Train and Test set in 80\%-20\% ratio where for every species 16 sequences were placed into Train Set and 4 sequences were placed into Test set. In cases where datasets were non-aligned, MAFFT 67] was used as multiple sequence alignemnt tool for aligning the barcode sequences. Most of the datasets were in FASTA format and a Python program was written in order to convert these FASTA files into corresponding CSV files. In case of a large number of missing nucleotide in barcode sequences of a species, the most frequently occurring certain nucleotide within that species was considered as replacement for that missing value. The related Python scripts written by us for converting necessary files to desired CSV format and for replacing missing values are available at https://github.com/MohimenulRafi/DNA_Barcode.git. 


\section{Experimental Arrangement}

For the purpose of species identification with DNA Barcode, we developed a Naïve Bayes classifier from scratch in Java fitting our methodological necessities (available at https://github.com/MohimenulRafi/DNA_Barcode.git). In the training phase, a probability matrix for each known species is calculated. For accomplishing this task, a Hashmap is used where the key is the species name and the value is the probability matrix. The matrix basically stores the probability of each nucleotide bases at each position in the Barcode sequence for a species. A worked-out example is demonstrated at Table 4 where it is considered that there are four different species and each having three different barcode sequences of length five. The resulting probability matrix of species 1 is shown in Table 5 .

Table 4. Sample Barcode Sequences of Four Species

\begin{tabular}{|c|c|c|c|}
\hline Species 1 & Species 2 & Species 3 & Species 4 \\
\hline ATTGC & TCCAG & GTATG & CGAAT \\
ATTCC & TCCGG & GCATG & CGGAT \\
ATTGC & TCCGC & GTATC & CGAAT \\
\hline
\end{tabular}

Table 5. Probability Matrix of Species 1

\begin{tabular}{|c|c|c|c|c|c|}
\hline Bases & Position 1 & Position 2 & Position 3 & Position 4 & Position 5 \\
\hline $\mathrm{A}$ & $3 / 3$ & $0 / 3$ & $0 / 3$ & $0 / 3$ & $0 / 3$ \\
$\mathrm{~T}$ & $0 / 3$ & $3 / 3$ & $3 / 3$ & $0 / 3$ & $0 / 3$ \\
$\mathrm{G}$ & $0 / 3$ & $0 / 3$ & $0 / 3$ & $2 / 3$ & $0 / 3$ \\
$\mathrm{C}$ & $0 / 3$ & $0 / 3$ & $0 / 3$ & $1 / 3$ & $3 / 3$ \\
\hline
\end{tabular}

In the test phase, for a query sequence, we calculate it's likelihood of being a particular species. Our model calculates the maximum likelihood and classify the new sequence to that corresponding species. For example, if we get a query sequence TCCAC from test set, our model takes the probability of T, C, C, A, C in position 1, 2, 3,4 and 5 respectively from probability matrix of species 1,2 and so on. Finally if the highest probability value considering the whole length of query sequence comes from the matrix of species 2, the sequence will be classified as species 2. Fig 1 shows an overview of this classification process.

To analyze the impact of length of DNA Barcode sequences on species identification, we have run our model with different sequence lengths on all the empirical as well as simulated datasets in a comprehensive manner.

\section{Results and Discussions}

After implementing our Naïve bayes classifier, we needed to test the effectiveness of our method. For this reason, to ensure the efficacy of our model, we ran our Naïve bayes classifier on first five of the COI gene based empirical datasets mentioned in Table 1 considering their full-length barcode sequences. After comparing the results with the maximum accuracy mentioned in other related studies [12] [4], where comparative performance analysis of different supervised machine learning approaches on those same COI based empirical datasets are provided, we thereby find our Naïve Bayes classifier competent with their performances (shown in Fig 2).

After testing our Naïve Bayes classifier on the COI based first five empirical datasets, subsequently we ran our model on all of the COI based empirical datasets 


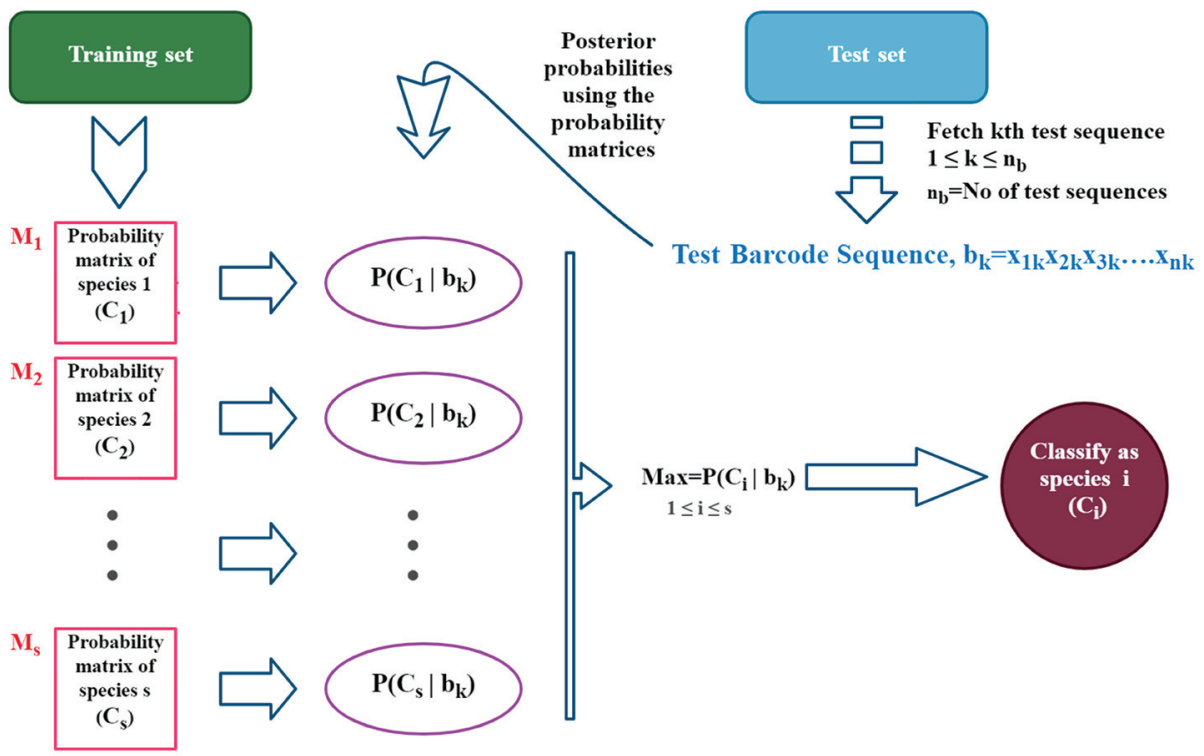

Fig 1. Overview of Species Identification with Naïve Bayes Procedure. Each test sequence is fetched from Test set and using the probability matrices the posterior probabilities are calculated. Based on the maximum probability score, that respective test sequence is classified to corresponding species label.

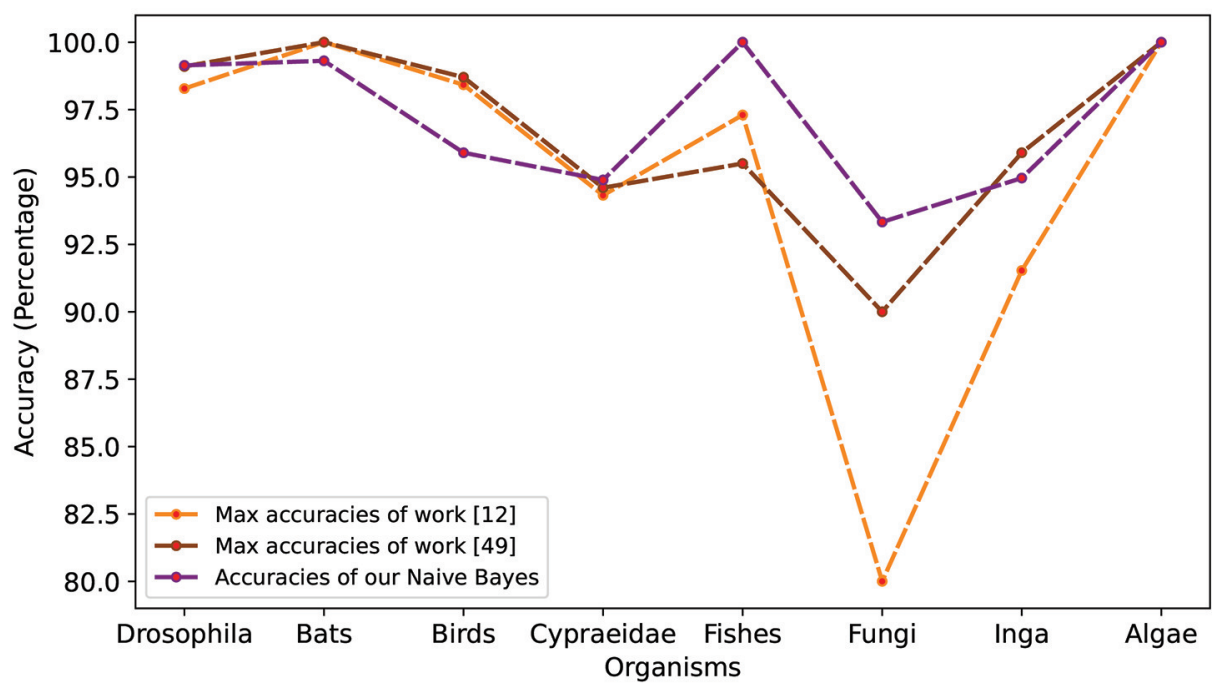

Fig 2. Performance Competency of our Naïve Bayes Classifier. The accuracy comparison of our model and the maximum accuracy score giving models of the works of [12] and [49] is depicted in this figure. Our model yields very competing performance in each cases.

mentioned in Table 1 with a view to analyzing accuracy responses varying barcode sequence lengths. As all of the datasets in Table 1 had barcode sequence lengths $>600$ bp commonly, we chose to set the upper bound of sequence length at 600 while varying lengths. Then the sequence lengths were decreasingly varied from this upper bound of $600 \mathrm{bp}$ with an interval of 50 bases and corresponding accuracy scores were calculated 
in each case. As 50 bp falls too low as sequence length, we considered 70 base-length after $100 \mathrm{bp}$ and tried to maintain this as the lower bound for varying sequence lengths throughout our study (with only a few exceptions). All these accuracy scores for varying DNA barcode lengths for COI based empirical datasets are represented in Table 6. These length-wise accuracy scores of Table 6 are also plotted in Fig 3 and demonstrated in the Heatmap of Fig 4 . If we analyze the pattern of these length-wise changes of accuracy scores in these plottings and heatmap, it seems that in most cases accuracy scores remain almost persistent in the range of 200bp-600bp. Although there is a gradual but trifling fall of accuracy in this range with the decrease of barcode sequence length, it significantly falls below 200bp barcode length.

Table 6. Accuracy Responses in COI based Empirical Dataset

\begin{tabular}{|c|c|c|c|c|c|c|c|c|c|c|c|c|}
\hline \multirow{2}{*}{ Datasets } & \multicolumn{12}{|c|}{ Lengths } \\
\hline & 70 & 100 & 150 & 200 & 250 & 300 & 350 & 400 & 450 & 500 & 550 & 600 \\
\hline Bats & 93.06 & 97.22 & | 99.31 & | $99.31 \mid$ & | 99.31 & $|99.31|$ & | 99.31 & $|99.31|$ & $|99.31|$ & $|99.31|$ & $|99.31|$ & | 99.31 \\
\hline Bird & 90.54 & 97.48 & 98.42 & | $98.42 \mid$ & | 98.42 & $|97.48|$ & |97.16 & $\mid 97.48$ & $|97.16|$ & $|97.16|$ & $|97.16|$ & |97.16 \\
\hline Cypraeidae & | 89.77 & 91.19 & 93.75 & |94.60| & | 94.03 & $|94.60|$ & | 95.17 & $|95.17|$ & $|95.17|$ & $|95.45|$ & $|95.45|$ & | 95.45 \\
\hline Drosophila & 96.55 & 98.28 & 98.28 & | 98.28 | & 98.28 & $|99.14|$ & $\mid 99.14$ & $|99.14|$ & $|99.14|$ & $|99.14|$ & $|99.14|$ & | 99.14 \\
\hline Fish & 88.29 & 93.69 & 99.1 & 100.0 & $\mid 100.0$ & $|100.0|$ & | 100.0 & $|100.0|$ & | $100.0 \mid$ & $|100.0|$ & $|100.0|$ & | 100.0 \\
\hline Butterfly & 89.8 & 93.47 & $\mid 95.92$ & |95.92| & $\mid 95.92$ & $|95.92|$ & $\mid 95.92$ & $\mid 95.92$ & $|95.92|$ & $|95.92|$ & $|96.33|$ & $\mid 96.33$ \\
\hline Bird 2 & 79.03 & 90.32 & 95.97 & | 96.37 | & 97.18 & $|97.18|$ & 97.58 & $|97.58|$ & $|98.79|$ & $|98.79|$ & $|98.79|$ & | 98.79 \\
\hline Butterfly 2 & 35.19 & 86.36 & 95.96 & |97.14| & | 97.14 & $|99.16|$ & | 99.49 & $|99.66|$ & $|99.66|$ & $|99.66|$ & $|99.66|$ & \begin{tabular}{|l}
99.66 \\
\end{tabular} \\
\hline Fish 2 & 45.92 & 84.69 & 89.8 & 91.84 & 91.84 & $|91.84|$ & 96.94 & $|96.94|$ & $|97.96|$ & $|97.96|$ & $|98.98|$ & | 98.98 \\
\hline
\end{tabular}
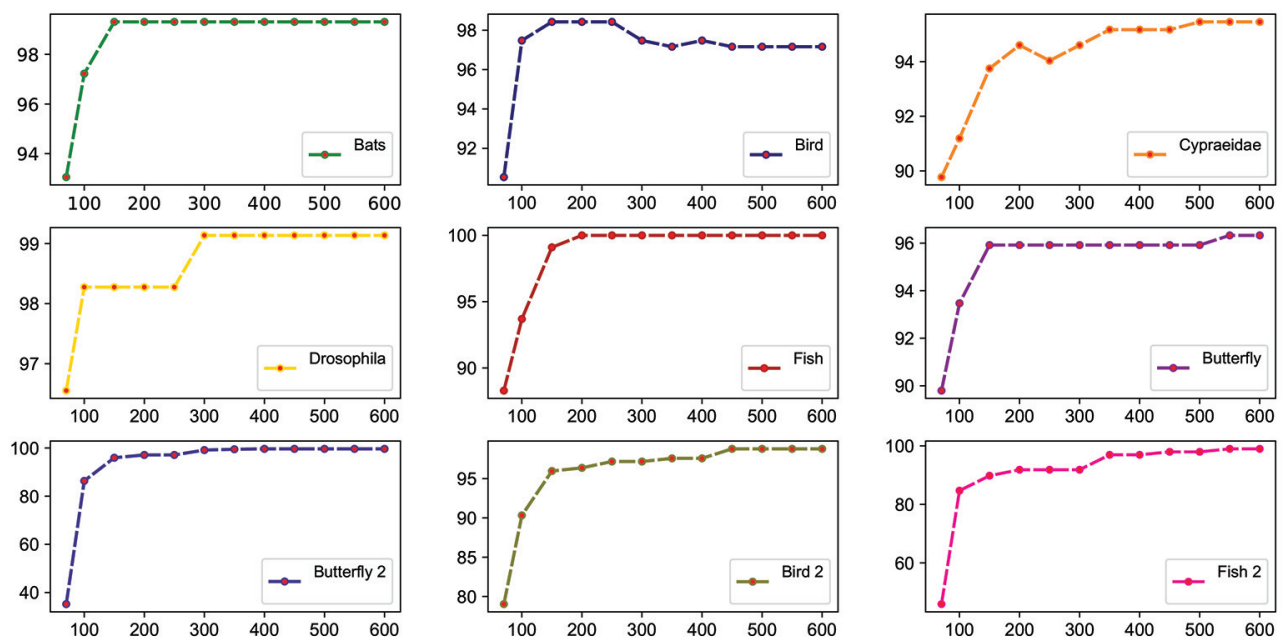

Fig 3. Lengthwise Accuracy comparison for COI based Empirical Datasets. From the lengthwise accuracy variations of each dataset, it is apparent that the accuracy is relatively consistent in the 200bp-600bp range but considerably falls below 200bp.

This similar approach is applied for the simulated datasets mentioned in Table 3 with maintaing same upper bound of $600 \mathrm{bp}$ and lower bound of 70bp. The length-wise accuracy responses for the simulated datasets are summarized in Table 7 and demonstrated in Fig 5. From this Fig 5 it is also evident that, the accuracy score drops 


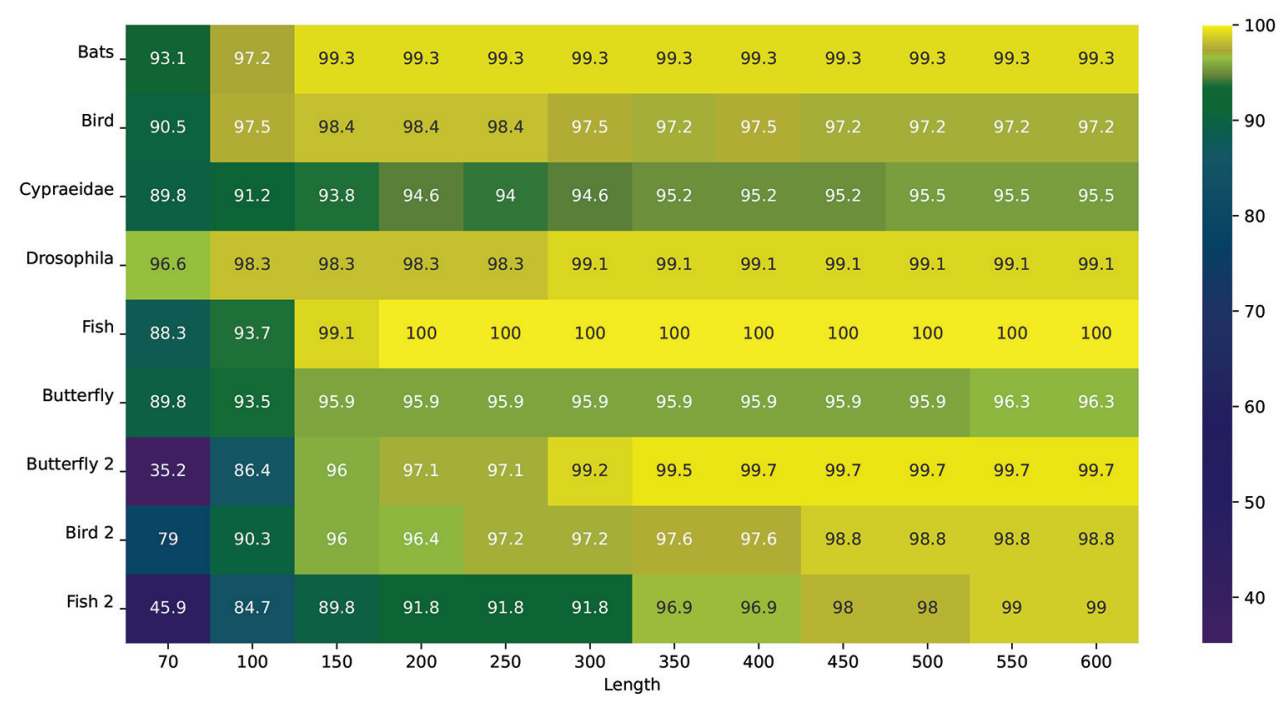

Fig 4. Heatmap of Lengthwise Accuracy Comparison for COI based Empirical Datasets. The lengthwise accuracy variation of Fig 3 is depicted in this heatmap. It is observed from the lengthwise color variation at each row that, 200bp-600bp provides a relatively consistent accuracy.

slightly and gradually with the reduction of sequence length and falls significantly when the sequence length is reduced to $<200 \mathrm{bp}$.

Table 7. Accuracy Responses in Simulated Datasets

\begin{tabular}{l|c|c|c|c|c|c|c|}
\hline \multirow{2}{*}{ Datasets } & \multicolumn{5}{|c|}{ Lengths } \\
\cline { 2 - 5 } & $\mathbf{7 0}|\mathbf{1 0 0}| \mathbf{1 5 0}|\mathbf{2 0 0}| \mathbf{2 5 0}|\mathbf{3 0 0}| \mathbf{3 5 0}|\mathbf{4 0 0}| \mathbf{4 5 0}|\mathbf{5 0 0}| \mathbf{5 5 0} \mid \mathbf{6 0 0}$ \\
\hline $\mathbf{N e 1 0 0 0}|75.91| 82.63|87.52| 90.34|91.88| 93.21|94.06| 94.84|95.26| 95.66|96.02| 96.32$ \\
\hline $\mathbf{N e 1 0 0 0 0}|81.05| 86.85|91.28| 93.21|94.43| 95.14|95.54| 95.84|96.08| 96.26|96.46| 96.60 \mid$ \\
\hline Ne50000 $|81.62| 85.80|88.65| 90.14|91.09| 91.56|91.99| 92.26|92.55| 92.73|92.92| 93.09 \mid$ \\
\hline
\end{tabular}

Furthermore, in order to get a complete overview of the effect of length on the species identification process, the datasets mentioned in Table 3 which are based on ITS and rbcL genes, are analyzed. For the dataset Fungi and Algae, we set upper bound and lower bound for varying sequence length as $850 \mathrm{bp}$ and $100 \mathrm{bp}$ respectively. For the dataset Inga, we set $1800 \mathrm{bp}$ and $1050 \mathrm{bp}$ as respective upper and lower bounds as the full lengths of barcode sequences in this dataset are $>1800 \mathrm{bp}$. In all three datasets, we decrease the length with an interval of 50 bases. The complete length-wise average accuracy analysis of these datasets is represented in Table 8 This analysis is also depicted in Fig 6 .

From there it can be seen that although the contexts are different from the previous length wise accuracy patterns of COI based empirical datasets and simulated datasets, still Algae and Fungi shows a sharp fall in accuracy scores around sequence lengths below 200-250bp. But this corroboration cannot be extended in case of Inga as its length-bounds had to be chosen differently; but it still shows a decline in accuracy score like every other ones if the lengths are gradually decreased from full length. In order to bring these three datasets on a common ground, average accuracy responses were further calculated at various percentage levels of their full lengths. The results are represented in Table 9 and depicted in Fig 7. From these representations, we can 


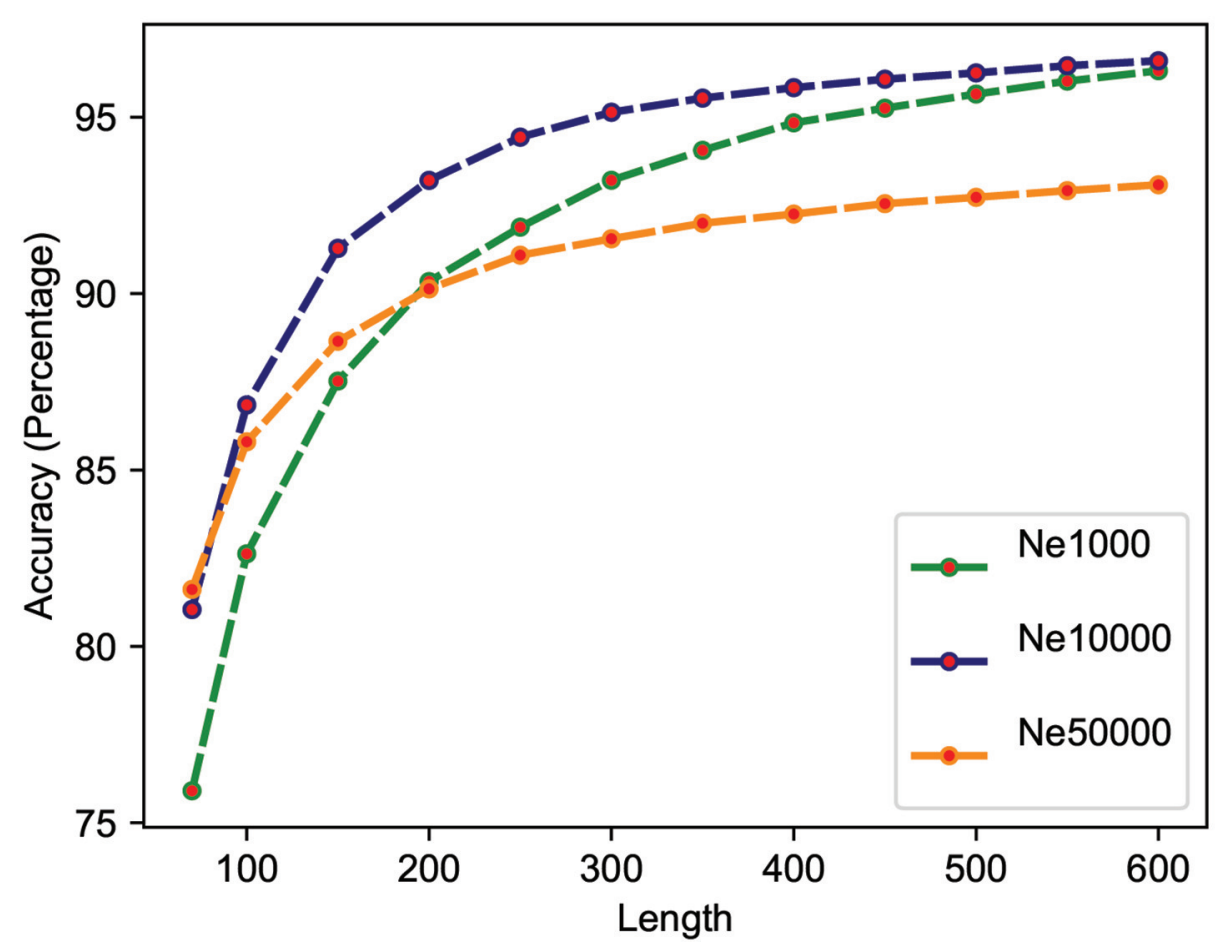

Fig 5. Lengthwise Accuracy comparison for Simulated Datasets. This figure suggests a gradual but minor drop in the accuracy score when reducing the sequence length from $600 \mathrm{bp}$ to $200 \mathrm{bp}$ whereas a comparatively significant fall is noticed when the sequence length is reduced below $200 \mathrm{bp}$.

Table 8. Lengthwise Accuracy Responses in Fungi, Inga and Algae Dataset

\begin{tabular}{|c|c|c|c|c|c|c|c|c|c|c|c|c|c|c|c|c|c|}
\hline & & \multicolumn{16}{|c|}{ Lengths } \\
\hline \multirow{2}{*}{ Fungi } & bp & 100 & 150 & 200 & 250 & 300 & 350 & 400 & 450 & 500 & 550 & 600 & 650 & 700 & 750 & 800 & 850 \\
\hline & $\%$ & 80.56 & | 88.89 | & $\mid 88.89$ & | 88.89| & $\mid 88.89$ & 88.89 & $\mid 88.89$ & 88.89 & | 88.89 & |88.89| & | $88.89 \mid$ & $|88.89|$ & | 88.89 | & 88.89 & 88.89 & | 88.89 \\
\hline \multirow{2}{*}{ Inga } & bp & 1050 & 1100 & 1150 & 1200 & 1250 & 1300 & 1350 & 1400 & 1450 & 1500 & 1550 & 1600 & 1650 & 1700 & 1750 & 1800 \\
\hline & $\%$ & 78.79 & $|78.79|$ & $\mid 78.79$ & $|78.91|$ & $\mid 85.09$ & 86.63 & $\mid 87.53$ & $\mid 87.40$ & $\mid 87.40$ & $|87.40|$ & | $87.14 \mid$ & | 89.71 & $|90.48|$ & 90.10 & |95.11 & $\mid 95.11$ \\
\hline \multirow{2}{*}{ Algae } & bp & 100 & 150 & 200 & 250 & 300 & 350 & 400 & 450 & 500 & 550 & 600 & 650 & 700 & 750 & 800 & 850 \\
\hline & $\%$ & 90.0 & 90.0 & 90.0 & 90.0 & 100.0 & $\mid 100.0$ & $\mid 100.0$ & $\mid 100.0$ & $\mid 100.0$ & $|100.0|$ & $|100.0|$ & $\mid 100.0$ & $\mid 100.0$ & $\mid 100.0$ & $\mid 100.0$ & | 100.0 \\
\hline
\end{tabular}

Table Notes: In this table, accuracy responses are demonstrated at different lengths for each of the Fungi, Inga and Algae datasets where respective results for each dataset consist of two rows. For each dataset, the first row indicates the lengths of the sequence (denoted by bp) and the second row indicates the corresponding accuracies (denoted by \%). 

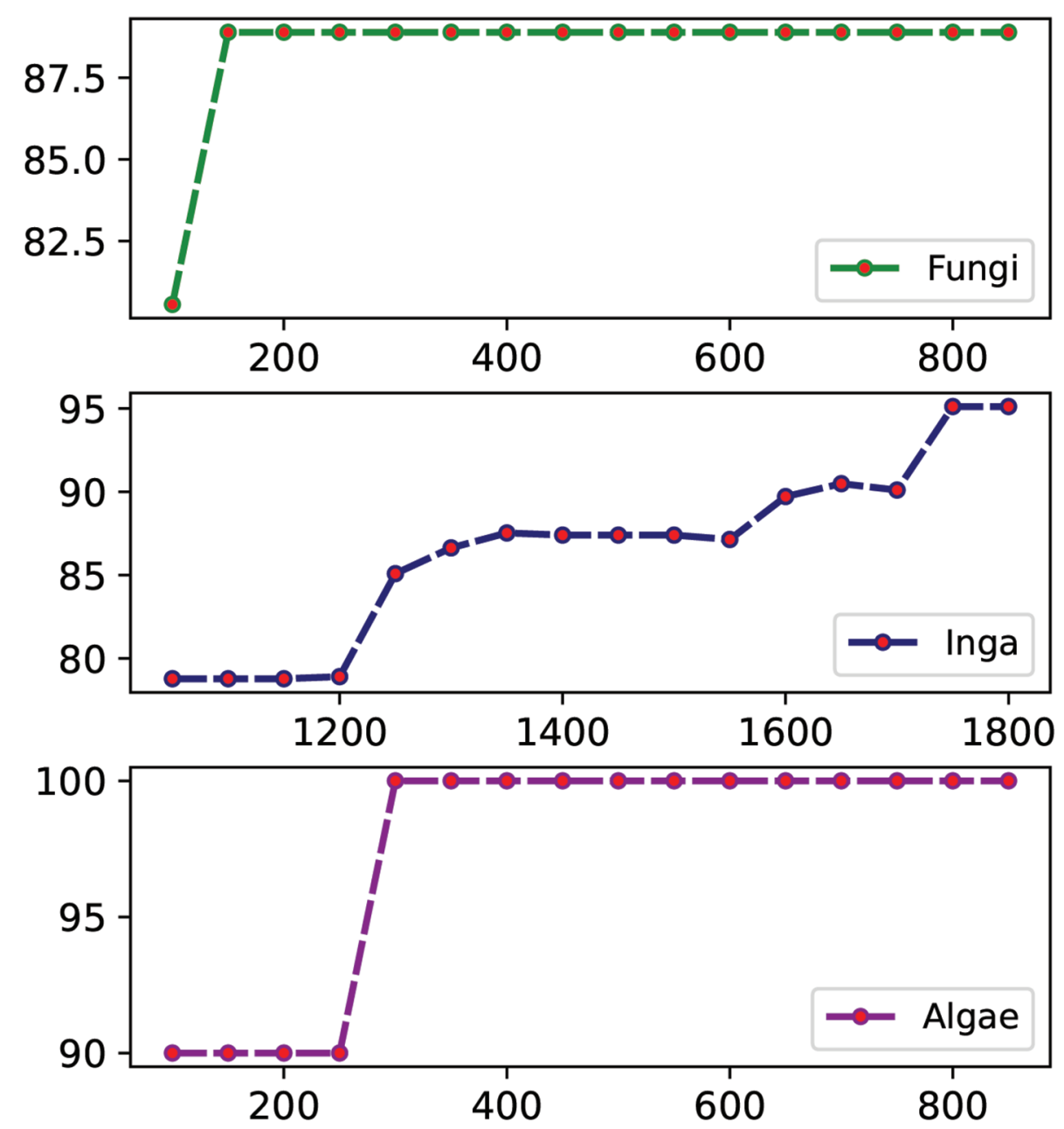

Fig 6. Lengthwise Accuracy comparison in Fungi, Inga and Algae Dataset. This figure demonstrates a sharp fall in accuracy scores for Algae and Fungi dataset around sequence lengths below 200bp-250bp. The length and subsequent length wise accuracy responses in Inga dataset are not similar to those of Fungi and Algae datasets, but still a decline in accuracy score can be observed with the gradual decrease of sequence length. 
observe that although no persistent range like previous COI based empirical datasets is evident from these ITS \& rbCL based datasets, the length-wise accuracy of Fungi and Algae falls sharply at around 20\%-30\% of their full lengths whereas the accuracy in Inga dataset considerably falls first at around $70 \%$ and later again at $40 \%$ of its full length. It is worth mentioning in this regard that, in case of COI based datasets and simulated datasets, the length ( $\sim 200 \mathrm{bp})$ below which a sharp decline was observed happens to be approx. $30 \%$ of the full length of barcode sequence. In both cases of Table 8 and Table 9. the first row denotes the length of the sequence and the second row denotes the corresponding accuracy considering that length respectively for each entry.

Table 9. Accuracy responses in Fungi, Inga and Algae dataset at different length-percentage

\begin{tabular}{|c|c|c|c|c|c|c|c|c|c|c|c|}
\hline & \multicolumn{10}{|c|}{ Percentages of Lengths } \\
\hline & & $10 \%$ & $20 \%$ & $30 \%$ & $40 \%$ & $50 \%$ & $60 \%$ & $70 \%$ & $80 \%$ & $|90 \%|$ & $100 \%$ \\
\hline \multirow{2}{*}{ Fungi } & bp & 93 & 186 & 279 & 372 & 465 & 558 & 651 & 744 & 837 & 930 \\
\hline & $\%$ & 79.44 & | 93.33 & 93.33 & 93.33 & | 93.33 & 93.33 & 93.33 & | 93.33 & |93.33 | & | 93.33 \\
\hline \multirow{2}{*}{ Inga } & bp & 183 & 367 & 551 & 735 & 919 & 1102 & 1286 & 1470 & 1654 & 1838 \\
\hline & $\%$ & 46.33 & | 66.84 & 67.09 & 76.41 & | 76.54 & 79.09 & 87.57 & | 88.49 & |90.06 | & 94.96 \\
\hline \multirow{2}{*}{ Algae } & bp & 112 & 225 & 338 & 451 & 564 & 676 & 789 & 902 & 1015 & 1128 \\
\hline & $\%$ & 90.0 & 90.0 & 100.0 & $\mid 100.0$ & $\mid 100.0$ & 100.0 & 100.0 & $\mid 100.0$ & 100.0 & 100.0 \\
\hline
\end{tabular}

Table Notes: In this table, accuracy responses are demonstrated at different percentage levels of the full sequence lengths for each of the Fungi, Inga and Algae datasets where respective results for each dataset consist of two rows. For each dataset, the first row indicates the lengths of the sequence (denoted by bp) at the mentioned percentage levels and the second row indicates the corresponding accuracies (denoted by \%).

In support of our findings we can allude to Fig 8 , which is adapted and redrawn from the work of [15]. In this figure, the proportion of species resolution with different DNA Barcode size is depicted. The shapes in individual plottings of Fig 3 also comport with the shape in Fig 8 implying the consistency between these separate but similar findings. In other related studies regarding mini-barcodes, it was also observed that full length barcodes provide marginally better identification success rates than mini barcodes having 200-400bp sequence length while the identification ability is significantly compromised only when the barcode length falls too short below 200bp [3]. In light of these above discussions, this proposition can be made that an approximation of 200-350bp mini-barcode length can be suggested for using in the process of species identification with DNA barcode approached with a supervised machine learning model where it has a significant possibility to perform with a reasonable accuracy score.

\section{Conclusion}

In this study, we tried to provide an approximation of required length of DNA Mini-barcode from the supervised learning perspective by analysing the length-wise accuracy in the species identification process. A Naïve Bayes classifier was implemented for the comprehensive analysis of the effect of varying DNA Barcode lengths on the identification accuracy. Our findings from this machine learning perspective bears consistency with the findings of other related biological works where DNA 


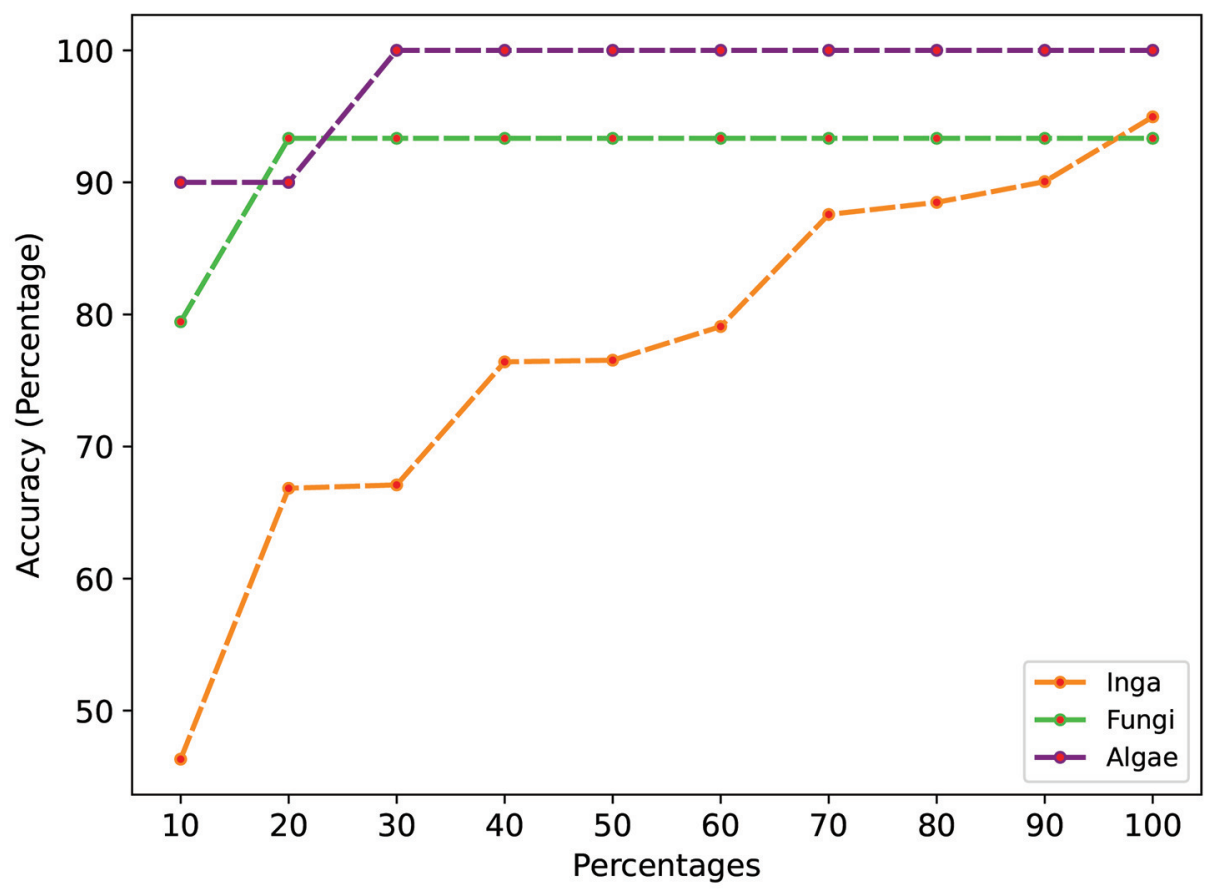

Fig 7. Accuracy responses in Fungi, Inga and Algae dataset at different length-percentage. As the sequence lengths of these three datasets are different, average accuracy responses were further calculated at various percentage levels of their full lengths to bring uniformity. 


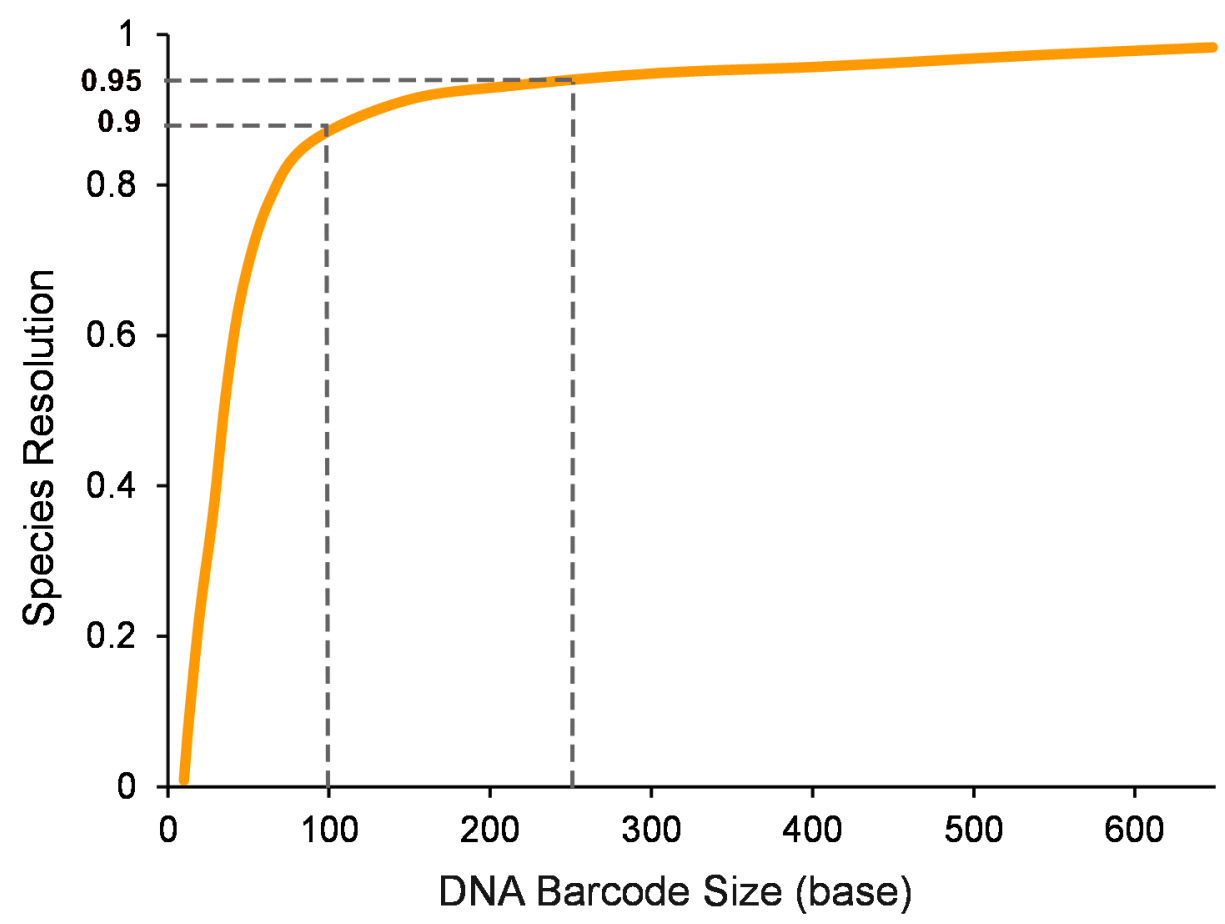

Fig 8. Change of Species Resolution in different DNA mini-barcode lengths (adapted and redrawn from [15]). In the demonstration of [15], it is asserted that although best performance can be achieved at full length barcode with $97 \%$ species resolution, $100 \mathrm{bp}$ and $250 \mathrm{bp}$ mini barcode provide $90 \%$ and $95 \%$ identification success respectively.

mini-barcodes were used and analyzed to facilitate the species identification process. Although we could perceive the approximate effective length of mini barcodes in case of COI gene based DNA sequences, further study is needed for plants and ITS gene barcode based organisms. Improving the quality of the sequence alignment by using better multiple sequence alignment tools/techniques might improve the identification accuracy in this regard. Other machine learning algorithms are also encouraged to be applied in this context to enrich the comprehensiveness of our finding.

\section{Acknowledgments}

We sincerely thank Md. Shamsuzzoha Bayzid for his valuable guidelines and continuous supports throughout the overall process.

\section{References}

1. Ruggiero MA, Gordon DP, Orrell TM, Bailly N, Bourgoin T, Brusca RC, et al. A higher level classification of all living organisms. PloS one. 2015;10(4):e0119248.

2. Mora C, Tittensor DP, Adl S, Simpson AG, Worm B. How many species are there on Earth and in the ocean? PLoS Biol. 2011;9(8):e1001127. 
3. Yeo D, Srivathsan A, Meier R. Longer is not always better: Optimizing barcode length for large-scale species discovery and identification. Systematic Biology. 2020;.

4. Dayrat B. Towards integrative taxonomy. Biological journal of the Linnean society. $2005 ; 85(3): 407-417$.

5. Armstrong K, Ball S. DNA barcodes for biosecurity: invasive species identification. Philosophical Transactions of the Royal Society B: Biological Sciences. 2005;360(1462):1813-1823.

6. Hebert PD, Cywinska A, Ball SL, Dewaard JR. Biological identifications through DNA barcodes. Proceedings of the Royal Society of London Series B: Biological Sciences. 2003;270(1512):313-321.

7. Hebert PD, Ratnasingham S, De Waard JR. Barcoding animal life: cytochrome c oxidase subunit 1 divergences among closely related species. Proceedings of the Royal Society of London Series B: Biological Sciences. 2003;270(suppl_1):S96-S99.

8. Group CPW, Hollingsworth PM, Forrest LL, Spouge JL, Hajibabaei M, Ratnasingham S, et al. A DNA barcode for land plants. Proceedings of the National Academy of Sciences. 2009;106(31):12794-12797.

9. Schoch CL, Seifert KA, Huhndorf S, Robert V, Spouge JL, Levesque CA, et al. Nuclear ribosomal internal transcribed spacer (ITS) region as a universal DNA barcode marker for Fungi. Proceedings of the National Academy of Sciences. 2012;109(16):6241-6246.

10. Weitschek E, Van Velzen R, Felici G, Bertolazzi P. BLOG 2.0: a software system for character-based species classification with DNA Barcode sequences. What it does, how to use it. Molecular ecology resources. 2013;13(6):1043-1046.

11. van Velzen R, Weitschek E, Felici G, Bakker FT. DNA barcoding of recently diverged species: relative performance of matching methods. PloS one. 2012;7(1):e30490.

12. Weitschek E, Fiscon G, Felici G. Supervised DNA Barcodes species classification: analysis, comparisons and results. BioData mining. 2014;7(1):1-18.

13. Hajibabaei M, Smith MA, Janzen DH, Rodriguez JJ, Whitfield JB, Hebert PD. A minimalist barcode can identify a specimen whose DNA is degraded. Molecular Ecology Notes. 2006;6(4):959-964.

14. Hajibabaei M, McKenna C. DNA mini-barcodes. In: DNA barcodes. Springer; 2012. p. 339-353.

15. Meusnier I, Singer GA, Landry JF, Hickey DA, Hebert PD, Hajibabaei M. A universal DNA mini-barcode for biodiversity analysis. BMC genomics. $2008 ; 9(1): 1-4$.

16. Staats M, Arulandhu AJ, Gravendeel B, Holst-Jensen A, Scholtens I, Peelen T, et al. Advances in DNA metabarcoding for food and wildlife forensic species identification. Analytical and Bioanalytical Chemistry. 2016;408(17):4615-4630.

17. Erickson DL, Reed E, Ramachandran P, Bourg NA, McShea WJ, Ottesen A. Reconstructing a herbivore's diet using a novel rbc L DNA mini-barcode for plants. AoB Plants. 2017;9(3):plx015. 
18. Frézal L, Leblois R. Four years of DNA barcoding: current advances and prospects. Infection, Genetics and Evolution. 2008;8(5):727-736.

19. Goldstein PZ, DeSalle R. Review and interpretation of trends in DNA barcoding. Frontiers in Ecology and Evolution. 2019;7:302.

20. Adamowicz SJ, Boatwright JS, Chain F, Fisher BL, Hogg ID, Leese F, et al. Trends in DNA barcoding and metabarcoding. Genome. 2019;62(3):v-viii.

21. Schindel DE, Miller SE. DNA barcoding a useful tool for taxonomists. Nature. 2005;435(7038):17-18.

22. Austerlitz F, David O, Schaeffer B, Bleakley K, Olteanu M, Leblois R, et al. DNA barcode analysis: a comparison of phylogenetic and statistical classification methods. BMC bioinformatics. 2009;10(S14):S10.

23. Casiraghi M, Labra M, Ferri E, Galimberti A, De Mattia F. DNA barcoding: a six-question tour to improve users' awareness about the method. Briefings in bioinformatics. 2010;11(4):440-453.

24. Little DP. DNA barcode sequence identification incorporating taxonomic hierarchy and within taxon variability. PLoS one. 2011;6(8):e20552.

25. Steinke D, Vences M, Salzburger W, Meyer A. TaxI: a software tool for DNA barcoding using distance methods. Philosophical Transactions of the Royal Society B: Biological Sciences. 2005;360(1462):1975-1980.

26. Ratnasingham S, Hebert PD. BOLD: The Barcode of Life Data System (http://www. barcodinglife. org). Molecular ecology notes. 2007;7(3):355-364.

27. Little DP, Stevenson DW. A comparison of algorithms for the identification of specimens using DNA barcodes: examples from gymnosperms. Cladistics. $2007 ; 23(1): 1-21$.

28. Altschul SF, Gish W, Miller W, Myers EW, Lipman DJ. Basic local alignment search tool. Journal of molecular biology. 1990;215(3):403-410.

29. Pearson W. Rapid and sensitive sequence comparison with FASTP and FASTA. Methods in enzymology. 1990;183:63-98.

30. Meier R, Shiyang K, Vaidya G, Ng PK. DNA barcoding and taxonomy in Diptera: a tale of high intraspecific variability and low identification success. Systematic biology. 2006;55(5):715-728.

31. Munch K, Boomsma W, Willerslev E, Nielsen R. Fast phylogenetic DNA barcoding. Philosophical Transactions of the Royal Society B: Biological Sciences. 2008;363(1512):3997-4002.

32. Saitou N, Nei M. The neighbor-joining method: a new method for reconstructing phylogenetic trees. Molecular biology and evolution. 1987;4(4):406-425.

33. Farris JS. Estimating phylogenetic trees from distance matrices. The American Naturalist. 1972;106(951):645-668.

34. Guindon S, Gascuel O. A simple, fast, and accurate algorithm to estimate large phylogenies by maximum likelihood. Systematic biology. 2003;52(5):696-704.

35. Huelsenbeck JP, Ronquist F. MRBAYES: Bayesian inference of phylogenetic trees. Bioinformatics. 2001;17(8):754-755. 
36. Abdo Z, Golding GB. A step toward barcoding life: a model-based, decision-theoretic method to assign genes to preexisting species groups. Systematic biology. 2007;56(1):44-56.

37. Pons J, Barraclough TG, Gomez-Zurita J, Cardoso A, Duran DP, Hazell S, et al. Sequence-based species delimitation for the DNA taxonomy of undescribed insects. Systematic biology. 2006;55(4):595-609.

38. Munch K, Boomsma W, Huelsenbeck JP, Willerslev E, Nielsen R. Statistical assignment of DNA sequences using Bayesian phylogenetics. Systematic biology. 2008;57(5):750-757.

39. Matz MV, Nielsen R. A likelihood ratio test for species membership based on DNA sequence data. Philosophical Transactions of the Royal Society B: Biological Sciences. 2005;360(1462):1969-1974.

40. Sarkar IN, Planet PJ, Desalle R. CAOS software for use in character-based DNA barcoding. Molecular Ecology Resources. 2008;8(6):1256-1259.

41. DasGupta B, Konwar KM, Măndoiu I, Shvartsman AA. DNA-BAR: distinguisher selection for DNA barcoding. Bioinformatics. 2005;21(16):3424-3426.

42. Chu KH, Xu M, Li CP. Rapid DNA barcoding analysis of large datasets using the composition vector method. BMC bioinformatics. 2009;10(S14):S8.

43. Kuksa P, Pavlovic V. Efficient alignment-free DNA barcode analytics. BMC bioinformatics. 2009;10(S14):S9.

44. Nugent CM, Adamowicz SJ. Alignment-free classification of COI DNA barcode data with the Python package Alfie. Metabarcoding and Metagenomics. 2020;4:e55815.

45. Albu M, Nikbakht H, Hajibabaei M, Hickey DA. The DNA barcode linker. Molecular ecology resources. 2011;11(1):84-88.

46. Singer GA, Hajibabaei M. iBarcode. org: web-based molecular biodiversity analysis. In: BMC bioinformatics. vol. 10. Springer; 2009. p. S14.

47. Lim J, Kim SY, Kim S, Eo HS, Kim CB, Paek WK, et al. BioBarcode: a general DNA barcoding database and server platform for Asian biodiversity resources. In: BMC genomics. vol. 10. Springer; 2009. p. S8.

48. Smagala JA, Dawson ED, Mehlmann M, Townsend MB, Kuchta RD, Rowlen KL. ConFind: a robust tool for conserved sequence identification. Bioinformatics. $2005 ; 21(24): 4420-4422$.

49. Kabir T, Shemonti AS, Rahman AH. Species Identification Using Partial DNA Sequence: A Machine Learning Approach. In: 2018 IEEE 18th International Conference on Bioinformatics and Bioengineering (BIBE). IEEE; 2018. p. 235-242.

50. He T, Jiao L, Wiedenhoeft AC, Yin Y. Machine learning approaches outperform distance-and tree-based methods for DNA barcoding of Pterocarpus wood. Planta. 2019;249(5):1617-1625.

51. Meher PK, Sahu TK, Gahoi S, Tomar R, Rao AR. funbarRF: DNA barcode-based fungal species prediction using multiclass Random Forest supervised learning model. BMC genetics. 2019;20(1):2. 
52. Meher PK, Sahu TK, Rao A. Identification of species based on DNA barcode using k-mer feature vector and Random forest classifier. Gene. 2016;592(2):316-324.

53. Kusuma WA, Nurilmala M, et al. Identification of Tuna and Mackerel Based on DNA Barcodes using Support Vector Machine (SVM). Telkomnika. 2016;14(2):778.

54. Anderson MP, Dubnicka SR. A sequential naive Bayes classifier for DNA barcodes. Statistical applications in genetics and molecular biology. 2014;13(4):423-434.

55. Porter TM, Gibson JF, Shokralla S, Baird DJ, Golding GB, Hajibabaei M. Rapid and accurate taxonomic classification of insect (class Insecta) cytochrome $\mathrm{c}$ oxidase subunit 1 (COI) DNA barcode sequences using a naïve Bayesian classifier. Molecular Ecology Resources. 2014;14(5):929-942.

56. Hajibabaei M, Janzen DH, Burns JM, Hallwachs W, Hebert PD. DNA barcodes distinguish species of tropical Lepidoptera. Proceedings of the National Academy of Sciences. 2006;103(4):968-971.

57. Lavinia PD, Núñez Bustos EO, Kopuchian C, Lijtmaer DA, García NC, Hebert PD, et al. Barcoding the butterflies of southern South America: Species delimitation efficacy, cryptic diversity and geographic patterns of divergence. PloS one. 2017;12(10):e0186845.

58. Meyer CP, Paulay G. DNA barcoding: error rates based on comprehensive sampling. PLoS biol. 2005;3(12):e422.

59. Lou M, Golding GB. Assigning sequences to species in the absence of large interspecific differences. Molecular Phylogenetics and Evolution. 2010;56(1):187-194.

60. Bertolazzi P, Felici G, Weitschek E. Learning to classify species with barcodes. BMC bioinformatics. 2009;10(S14):S7.

61. Hebert PD, Stoeckle MY, Zemlak TS, Francis CM. Identification of birds through DNA barcodes. Plos biol. 2004;2(10):e312.

62. Kerr KC, Stoeckle MY, Dove CJ, Weigt LA, Francis CM, Hebert PD. Comprehensive DNA barcode coverage of North American birds. Molecular ecology notes. 2007;7(4):535-543.

63. Ward RD, Zemlak TS, Innes BH, Last PR, Hebert PD. DNA barcoding Australia's fish species. Philosophical Transactions of the Royal Society B: Biological Sciences. 2005;360(1462):1847-1857.

64. Dexter KG, Pennington TD, Cunningham CW. Using DNA to assess errors in tropical tree identifications: How often are ecologists wrong and when does it matter? Ecological Monographs. 2010;80(2):267-286.

65. Rish I, et al. An empirical study of the naive Bayes classifier. In: IJCAI 2001 workshop on empirical methods in artificial intelligence. vol. 3; 2001. p. 41-46.

66. Denver DR, Morris K, Lynch M, Vassilieva LL, Thomas WK. High direct estimate of the mutation rate in the mitochondrial genome of Caenorhabditis elegans. Science. 2000;289(5488):2342-2344. 
bioRxiv preprint doi: https://doi.org/10.1101/2020.12.10.420281; this version posted December 22, 2020. The copyright holder for this preprint (which was not certified by peer review) is the author/funder. All rights reserved. No reuse allowed without permission.

67. Katoh K, Standley DM. MAFFT multiple sequence alignment software version 7: improvements in performance and usability. Molecular biology and evolution. $2013 ; 30(4): 772-780$. 